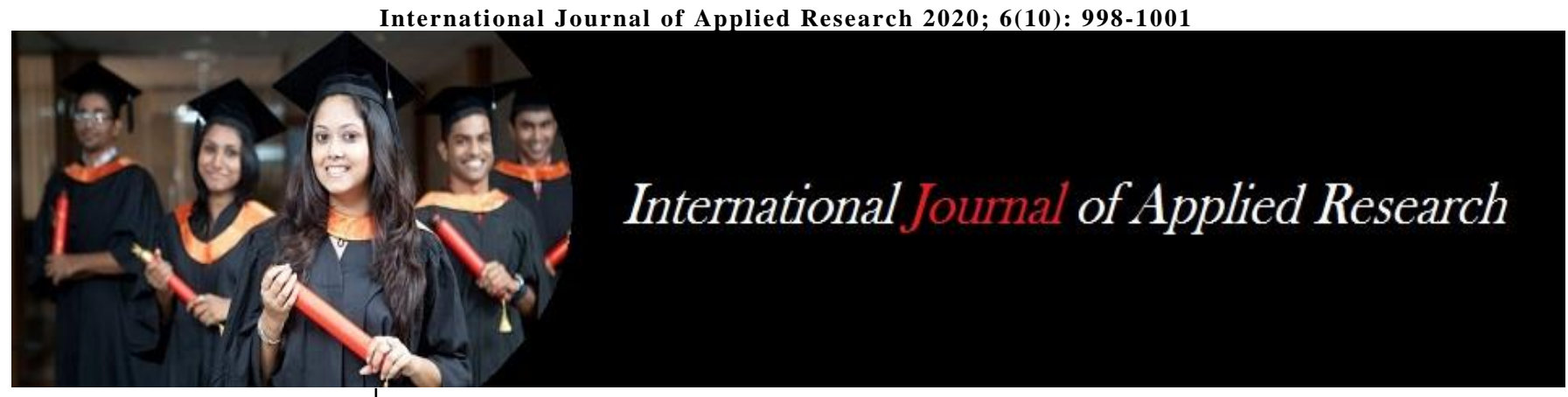

ISSN Print: 2394-7500 ISSN Online: 2394-5869 Impact Factor: 8.4 IJAR 2020; 6(10): 998-1001 www.allresearchjournal.com Received: 16-08-2020 Accepted: 19-09-2020

\section{Adama Dembélé}

Laboratoire de chimie organique et substances naturelles, Faculté des sciences et Techniques (FST), Université des Sciences, des Techniques et des Technologies de Bamako (USTTB), Bamako, Mali

\section{Lassana Sissoko}

Département de Médecine

Traditionnelle, Institut National en Santé Publique, Bamako, Mali

\section{Issiaka Togola}

Laboratoire de Biochimie Végétale Alimentaire et de Biotechnologie (LBVAB), Faculté des Sciences et Techniques, Université des Sciences, des Techniques et des Technologies de Bamako, Bamako, Mali

\section{Nah Traoré}

Laboratoire de chimie organique et substances naturelles, Faculté des sciences et Techniques (FST), Université des Sciences, des Techniques et des Technologies de Bamako (USTTB), Bamako, Mali

\section{Lassine Sidibé}

Laboratoire de chimie organique et substances naturelles, Faculté des sciences et Techniques (FST), Université des Sciences, des Techniques et des Technologies de Bamako (USTTB), Bamako, Mali

Drissa Diallo

Département de Médecine Traditionnelle, Institut National en Santé Publique, Bamako, Mali

Corresponding Author: Issiaka Togola

Laboratoire de Biochimie Végétale, Alimentaire et de Biotechnologie (LBVAB), Faculté des Sciences et Techniques, Université des Sciences, des Techniques et des Technologies de Bamako, Bamako, Mali

\section{Enquête ethnobotanique de trois plantes utilisées dans la prise en charge traditionnelle de l'hypertension artérielle au Mali: Balanites aegyptiaca (L.) Del., Piliostigma reticulatum (DC), Hochst et Bauhinia rufescens (L.)}

\author{
Adama Dembélé, Lassana Sissoko, Issiaka Togola, Nah Traoré, Lassine \\ Sidibé and Drissa Diallo
}

DOI: https://doi.org/10.22271/allresearch.2020.v6.i10o.7683

\begin{abstract}
Notre travail a porté sur l'étude ethnobotanique des trois plantes (Balanites aegyptiaca (L.) Del., Piliostigma reticulatum (DC), Hochst, Bauhinia rufescens L.) utilisées par les tradipraticiens pour le traitement de l'hypertension artérielle. Ainsi, une enquête ethnobotanique a été menée dans les villes de Siby, Kolokani, Ségou et Bamako au Mali. Au total 105 tradi-praticiens de santé ont été interrogé dont la majorité était des hommes avec $75,96 \%$ contre $24,04 \%$ de femmes et l'âge moyen était de 54 ans. En sommes, 18 recettes monospécifiques et/ou plurispécifiques ont été recensées dans ces quatre localités et le mode d'administration des préparations à base de ces plantes reste oral. Ces résultats constituent une bonne base de données pour des investigations phytochimiques et des criblages biologiques dans la recherche de molécules anti-hypertensives à base des plantes.
\end{abstract}

Keywords: Ethnobotanique, Hypertension artérielle, Plantes médicinales, Mali

\section{Introduction}

Les pays sont confrontés à l'émergence de l'hypertension artérielle qui devient de plus en plus un véritable problème de santé publique. En effet, la prévalence de la maladie en Afrique subsaharienne est élevée chez les adultes âgés de 18 ans et plus, allant de $16 \%$ à 40 $\%$. Dans certaines études, la prévalence dépasse $60 \%$ chez les personnes âgées de 65 ans et plus (Houehanou et al., 2018) ${ }^{[1]}$. Au Mali, cette prévalence varie selon les études de 20,83\% à 39,4\% (Menta et al., 2018) ${ }^{[2]}$. Cette maladie peut causer de graves dommages au niveau des artères cérébrales, cardiaques et rénales, favorisant l'apparition de complications graves, telles que les accidents vasculaires cérébraux, l'infarctus du myocarde et les insuffisances cardiaques ou rénales (Goïta et al., 2020) ${ }^{[3]}$. De nombreux traitements par des molécules de synthèse sont actuellement proposés pour pallier à ces troubles mais leur capacité à provoquer des effets secondaires indésirables redonnent un nouvel élan aux substances naturelles (Akassa et al., 2019) ${ }^{[4]}$. Par ailleurs, le coût élevé des prestations de santé et des médicaments ainsi que les facteurs socio-économiques poussent une grande partie de la population à utiliser les plantes médicinales pour se soigner (Sanogo, 2014) ${ }^{[5]}$. Ainsi, au Mali, $80 \%$ de la population ont recours à la médecine traditionnelle pour traiter de nombreuses maladies. Diallo et al., (2001) ${ }^{[6]}$. Balanites aegyptiaca (L.) Del., Piliostigma reticulatum (DC), Hochst, et Bauhinia rufescens Lam font parties des plantes les plus utilisées dans la prise en charge de l'hypertension au Mali. L'objectif du présent travail est de contribuer à une meilleure connaissance des plantes utilisées dans la prise en charge de l'hypertension.

\section{Méthodologie}

Du 13 juin 2006 au 27 juillet 2006, nous avons mené une étude sur les connaissances, attitudes et pratiques des tradipraticiens de santé (TPS) face à l'hypertension artérielle dans quatre localité du Mali; Kolokani (région de Koulikoro), Siby (région de Koulikoro), cercle 
de Ségou (région de Ségou) et le district de Bamako (Lafiabougou, Hamdalaye, Djicoroni Para, Sébénikoro, Kalabancoura, Moussabougou). L'enquête a été réalisée par des entretiens individuels (Diarra et al., 2016; Somboro et al., 2011) ${ }^{[7,8]}$. Les TPS reconnus par la population ont été identifiés grâce à la collaboration de leur fédération FEMATH (Fédération Malienne des Associations de Thérapeutes et Herboristes).

Les questions portaient sur l'énumération des symptomatologies de l'hypertension artérielle, les parties de trois plantes Balanites aegyptiaca (L.) Del., Piliostigma reticulatum (DC), Hochst, et Bauhinia rufescens Lam (feuilles, racines, écorce du tronc, fruits) utilisées, les modes de préparation (décoction, macération) et les modes d'administration des recettes.

\section{Analyse des données}

Le logiciel Excel 2010 a servi à analyser les données issues de l'enquête.

\section{Résultats Et Discussions}

\section{Données sociodémographiques}

Cette étude a enrôlé au total 105 tradi-praticiens de santé (TPS) constitués de femmes et d'hommes répartie comme suit: 19 TPS à Kolokani, 21 à Siby, 25 à Ségou et 40 dans le district de Bamako. La figure 1 montre la répartition des TPS de chaque localité en fonction du sexe. Dans l'ensemble, nous avons enrôlé 24,04\% de femmes contre $75,96 \%$ d'hommes. Même beaucoup de travaux ont montré que les femmes les plus représentées dans cette activité (Samaké et al., 2020; Appiah et al., 2018; Kasolo et al., $2010)^{[9,10,11]}$; cette étude est en accord avec celle de (Compaore et al., 2020; Diarra et al., 2016; Gbekley et al., 2015) ${ }^{[12,7,13]}$. Aussi, la moyenne d'âge était de 54 ans et $75,85 \%$ des TPS avaient reçu leur savoir par le biais de l'héritage familial, tandis que $24,15 \%$ l'ont acquis par des recherches personnelles Ces phénomènes peuvent s'expliquer par le fait que dans la société africaine, les hommes sont privilégiés dans la transmission des connaissances sur le traitement des maladies en général et les femmes s'intéressent aux maladies infantiles (Zizka et al., 2015) $)^{[14]}$. Pour Gbekley et al., (2015) ${ }^{[13]}$, la connaissance d'une recette en médecine traditionnelle est avant tout un secret de famille qui est transmis de génération en génération par le biais des coutumes et de la tradition orale. En plus, la pratique de la médecine traditionnelle serait l'apanage des hommes d'âge mûr (Aburjai et al., 2007) ${ }^{[15]}$.

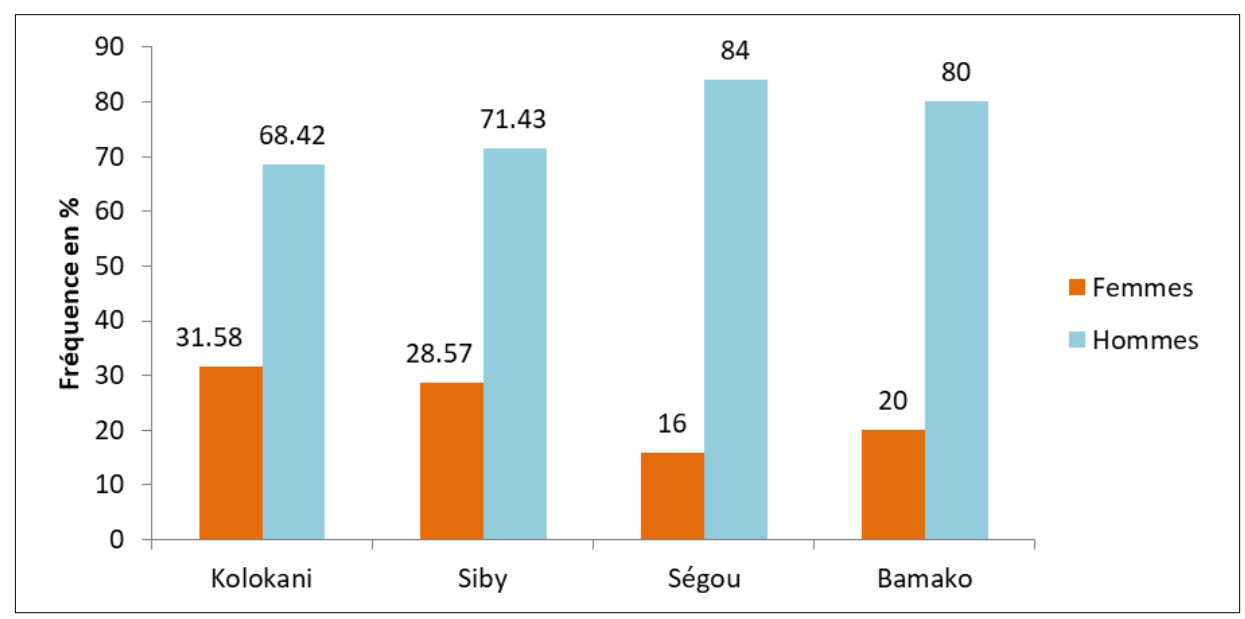

Fig 1: Répartition des tradi-thérapeutes en fonction du sexe et par localité

\section{Données ethnobotaniques}

L'hypertension artérielle a plusieurs appellations, nous avons noté entre autres, "koo tana dyankaro"' (Malinké), qui signifie maladie du sel, djolithiaya bana (Bambara) ou maladie qui augmente le sang, "daha dyankaro"' (Malinké) ou maladie des riches. Les expressions 'tason dyima', et tension yèlèbana sont aussi utilisé pour désigner la maladie, termes empruntés à la médecine moderne qui signifie l'hypertension. Les symptomatologies les plus citées dans les quatre localités sont les vertiges, le bourdonnement d'oreilles, le fourmillement des membres. Les traitements étaient surtout à base de substances végétales et les feuilles sont citées dans la plupart des cas. Ainsi, 18 recettes avec Balanites aegyptiaca (L.) Del., Piliostigma reticulatum (DC), Hochst, et Bauhinia rufescens Lam. ont été proposés pour le traitement de l'hypertension artérielle.

\section{Balanites aegyptiaca (Linn).Del; Nsègènè (Bambara), Sègènè (Malinké)}

1. Boire le macéré de deux verres à café (environ $200 \mathrm{ml}$ ) de la poudre de feuilles de Balanites aegyptiaca, 3 fois par jour après les repas, matin, midi, soir, pendant deux semaines. Le traitement comporte un régime sans sel et sans graisse.

2. Préparer un décocté de deux litres de trois bottes de feuilles de Balanites aegyptiaca. Prendre deux verres à café trois fois par jour jusqu'à guérison.

3. Faire une macération dans un $1 / 2$ litre d'eau de trois pincées à cinq doigts de la poudre de feuilles de Balanites aegyptiaca. Boire le macéré à volonté pendant une semaine, éviter les repas salés et huileux, contrôler la tension régulièrement après la prise des médicaments.

4. Préparer le macéré d'un bol d'eau de deux poignées $(2 \times 60 \mathrm{~g})$ de la poudre de feuilles de Balanites aegyptiaca associé à une poignée de poudre de feuilles de Sula finsan (Trichilia roka Chiov.). Consommer deux verres à café du macéré deux fois par jour, matin et soir, pendant trois semaines.

5. Sécher et pulvériser les feuilles de Balanites aegyptiaca. Consommer la poudre, trois cuillérées à café deux fois par jour matin et soir. Eviter les excitants, les repas huileux et salés. 
6. Macérer dans l'eau la poudre de trois poignées $(3 \times 60 \mathrm{~g})$ pour les hommes, quatre poignées $(4 \times 60 \mathrm{~g})$ pour les femmes, des feuilles de Balanites aegyptiaca, de Bauhinia rufescens. Boire le macéré trois fois par jour, matin, midi, soir, après les repas pendant trois semaines et contrôler la tension.

7. Faire bouillir dans deux litres d'eau trois bottes de feuilles de Balanites aegyptiaca, de Bauhinia rufescens. Boire le décocté à volonté jusqu'à guérison.

\section{Bauhinia rufescens (Lam.); Geseme (Bambara), Sifile iri (Malinké)}

1. Bouillir dans l'eau trois bottes des feuilles de Bauhinia rufescens, deux bottes des feuilles de Combretum micranthum G. Don. Utiliser le décocté ainsi obtenu en substitution de l'eau de boisson pendant trois semaines.

2. Macérer trois poignées (environ $3 \times 60 \mathrm{~g}$ ) de poudre des feuilles de Bauhinia rufescens dans un litre d'eau pendant trois heures. Boire le macéré à volonté pendant deux à trois semaines, ce produit augmente le volume urinaire.

3. Macérer des feuilles pilées de Bauhinia rufescens dans une tasse contenant 1,5 litre d'eau pendant une nuit. Boire un bol (environ $750 \mathrm{ml}$ ) de ce macéré chaque jour après le déjeuner pendant deux semaines.

4. Macérer dans 1,5 litre d'eau trois poignées de poudre de feuilles, une poignée de poudre de racines, une poignée d'écorce de tronc de Bauhinia rufescens. Filtrer, boire le macéré matin et soir pendant un mois. Le traitement comporte un régime sans sel et pauvre en graisse.

5. Macérer un mélange à quantités égales de feuilles de Bauhinia rufescens et de Cassia occidentalis L. dans de l'eau pendant $24 \mathrm{~h}$. Recueillir le macéré et le boire au moins deux fois par jour pendant vingt jours.

6. Porter à ébullition dans l'eau trois bottes des feuilles de Bauhinia rufescens, deux bottes de feuilles de Cassia occidentalis L. Boire un bol $(750 \mathrm{ml})$ de ce décocté chaque jour après le déjeuner.

\section{Piliostigma reticulatum (D.C.) Hochst; Nyamacè (Bambara), Nyama ke (Malinké)}

1. Macérer de deux à quatre poignées de poudre de feuilles de Piliostigma reticulatum dans un récipient contenant d'eau (environ 1 litre) pendant 8 heures. Boire le macéré à volonté pendant trois semaines. Ce produit augmente le volume urinaire.

2. Bouillir dans l'eau trois bottes de feuilles Piliostigma reticulatum. Boire un à deux bols de ce décocté matin et soir, pendant 15 jours. Contrôler la tension après les 15 jours.

3. Macérer des feuilles pilées de Piliostigma reticulatum dans une calebasse contenant deux litres d'eaux pendant une nuit. Boire deux bols de ce macéré deux fois par jour matin et soir, après les repas. Eviter les repas salés et huileux; le traitement dure quatre semaines.

4. Sécher et pulvériser les feuilles de Piliostigma reticulatum. Consommer la poudre trois cuillérées à café pour les hommes et quatre cuillérées pour les femmes deux fois par jour.

5. Macérer un mélange à quantités égales des feuilles de Piliostigma reticulatum et de Trichilia roka Chiov. dans pendant une nuit. Boire le macéré à volonté deux fois par jour matin et soir. Eviter les repas salés et gras surtout les nuits, le traitement dure trois semaines.
L'utilisation de ces plantes dans la prise en charge des maladies cardiovasculaires ont été signalé dans de nombreux travaux (Dongock et al., 2018; Jazy et al., 2017; Nole et al., 2016) [16, 17, 18]. Certaines recettes sont monospécifiques tandis que d'autres sont préparées en association de plusieurs espèces; ce qui permettrait de créer une dynamisation des effets curatifs des divers constituants afin d'aboutir à une synergie thérapeutique (Bla et al., 2015) [19]. L'activité anti-hypertensive de certaines de ces plantes a également été prouvée expérimentalement par des études in vivo ou in vitro (Ighodaro et Omole, 2012; El Mastry et al., 2010) ${ }^{[20,21]}$. Dans cette étude l'administration des recettes se fait exclusivement per os. Cette prescription peut s'expliquer par le fait que la maladie (HTA) est liée à des organes profonds et pour les atteindre, tout composé doit transiter par l'appareil digestif pour en faciliter son assimilation (Tra Bi et al., 2008; Orch et al., 2015) ${ }^{[22,23] .}$

\section{Conclusion}

Il ressort de cette étude que Balanites aegyptiaca, Bauhinia rufescens et Piliostigma reticulatum constituent des plantes très importantes en milieu Malinké et Bambara dans la prise en charge de l'hypertension artérielle. Ces plantes sont préparées en recettes monospécifiques et/ou plurispécifiques par des tradi-praticiens de santé majoritairement de sexe masculin. L'administration de ces boissons à base de plantes se fait uniquement par voie orale.

\section{Référence}

1. Houehanou C, Amidou S, Preux PM, Houinato D, Lacroix P. Hypertension artérielle (HTA) en Afrique subsaharienne. JMV-Journal de Médecine Vasculaire 2018,43(2).

2. Menta I, Ba HO, Sanogo KM. Hypertension (HTA) among young people of 18 to 35 years old in cardiology department of Gabriel Touré university teaching hospital. World Journal of Cardiovascular Diseases 2018;8(1):11-17.

3. Goïta Y, Chao de la Barca JM, Keita A, Diarra MB, Dembélé KC, Dramé BSI et al. Analyse biochimique multi-paramétrique révélant une augmentation de l'homocystéinémie et du NT-ProBNP chez les patients hypertendus à Bamako (Mali). Pan African Medical Journal. 2020;35:10. doi:10.11604/pamj.2020.35.10.18821.

4. Akassa H, Ondele R, Peneme BML, Etou Ossibi AW, Morabandza CJ, Tamboura HH. Activité aphrodisiaque et étude du mécanisme d'action de l'extrait aqueux des écorces de tronc de Pausinystalia yohimbe Kschum (Rubiaceae) chez le rat wistar. Journal of Animal \& Plant Sciences 2019;9(1):6372-6383.

5. Sanogo R. Pteolopsis suberosa Engl. Et Diels (Combretaceae): une plante à activité antiulcère et anti Helicobacter pylori. Hegel 2014;4(2):148-153. DOI: 10.4267/2042/53781.

6. Diallo D, Marston A, Terreaux C, Touré Y, Smestad Paulsen B, Hostettmann K. Screening of malian medicinal plants for antifungal, larvicidal, molluscicidal, antioxidant and radical scavenging activities. Phytother. Res 2001:15(5):401-406.

7. Diarra N, Togola A, Denou A, Merlin Willcox M, Daou C. et Diallo D. Etude ethnobotanique des plantes alimentaires utilisées en période de soudure dans les régions sud du Mali. Int. J Biol. Chem. Sci 
2016;10(1):184-197.

http://dx.doi.org/10.4314/ijbcs.v10i1.14

8. Somboro AA, Patel K, Diallo D, Sidibe L, Chalchat JC, Figueredo G. An ethnobotanical and phytochemical study of the African medicinal plant guiera senegalensis J.F. Gmel. Journal of Medicinal Plants Research 2011;5(9):1639-1651.

9. Samaké S, Togola I, Diarra N, Samaké M, Sanogo R. Etude ethnobotanique et screening phytochimique de plantes ornementales à usage thérapeutique du district de Bamako, Mali. Int. J Adv. Res 2020;8(08):251-262. http://dx.doi.org/10.21474/IJAR01/11487.

10. Appiah KS, Oppong CP, Mardan HK, Omari RA, Kpabitey S, Amoatey CA et al. Medicinal Plants Used int he Ejisu-Juaben Municipality, Southern Ghana: An Ethnobotanical Study. Medicine 2018;6:1. doi:10.3390/medicines6010001.

11. Kasolo JN, Bimenya GS, Ojok L, Ochieng J, OgwalOkeng JW. Phytochemicals and uses of Moringa oleifera leaves in Ugandan rural communities. Journal of Medicinal Plants Research 2010;4:753-757.

12. Compaore S, Belemnaba L, Koala M, Magnini RD, Ouedraogo N, Thiombiano A. Consensus level in the traditional management of diabetes and chemical potentiality of plants from north Sudanese, Burkina Faso. J Med. Plants Res 2020;14(8):415-427. DOI: 10.5897/JMPR2020.6967.

13. Gbekley EH, Karou DS, Gnoula C, Agbodeka K, Anani $\mathrm{K}$, Tchacondo $\mathrm{T}$ et al. Étude ethnobotanique des plantes utilisées dans le traitement du diabète dans la médecine traditionnelle de la région Maritime du Togo. Pan African Medical Journal. 2015;20:437. doi:10.11604/pamj.2015.20.437.5660.

14. Zizka A, Thiombiano A, Dressler S, Nacoulma B, Ouédraogo A, Ouédraogo I et al. Traditional plant use in Burkina Faso (West Africa): A national-scale analysis with focus on traditional medicine. Journal of Ethnobiology and Ethnomedicine 2015;11:1-34.

15. Aburjai T, Hudaib M, Tayyem R, Yousef M, Qishawi M. Ethnopharmacological survey of medicinal herbs in Jordan, the Ajloun Heights region. J Ethnopharmacol. 2007;110(2):294-304.

16. Dongock DN, Bonyo AL, Mapongmestem PM, Bayegone E. Etude ethnobotanique et phytochimique des plantes médicinales utilisées dans le traitement des maladies cardiovasculaires à Moundou (Tchad). Int. J Biol. Chem. Sci 2018;12(1):203-216.

17. Jazy MA, Karim S, Morou B, Snogo R, et Mahamane $\mathrm{S}$. Enquête ethnobotanique auprès des tradipraticiens de santé des régions de Niamey et Tillabéri au Niger: données 2012-2017. European Scientific Journal. 2017;13(33):276-304. http://dx.doi.org/10.19044/esj.2017.v13n33p276

18. Nole T, Lionel TDW, Cedrix TFS, Gabriel AA. Ethnomedical and Ethnopharmacological Study of Plants Used For Potential Treatments of Diabetes and Arterial Hypertension by Indigenous People in Three Phytogeographic Regions of Cameroon. Diabetes Case Reports 2016;1:110.

19. Bla KB, Trebissou JND, Bidie A. Étude ethnopharmacologique des plantes antipaludiques utilisées chez les Baoulé-N'Gban de Toumodi dans le Centre de la Côte d'Ivoire. J Appl. Biosc 2015;85:77757783 .
20. Ighodaro OM, Omole JO. Effects of Nigerian Piliostigma thonningii Species Leaf Extract on Lipid Profile in Wistar Rats. ISRN Pharmacology 2012;(2):387942. doi:10.5402/2012/387942.

21. El Mastry SM, Ebeed MM, El Sayed IH, Nasr MY, El Halafawy KA. Protective effect of Balanites aegyptiaca on antioxidant defense system against adriamycininduced cardiac toxicity in expermental mice. Egypt $\mathrm{J}$ Biochem Mole Biol 2010;28:1. http://ajol.info/index.php/ejbmb/article/view/54368.

22. Tra Bi FH, Irié GM, N'gaman KCC, Mohou CHB. Études de quelques plantes thérapeutiques utilisées dans le traitement de l'hypertension artérielle et du diabète: deux maladies émergentes en Côte d'Ivoire. Sciences \& Nature 2008;5(1):39-48.

23. Orch H, Douira A, et Zidane L. Étude ethnobotanique des plantes médicinales utilisées dans le traitement du diabète, et des maladies cardiaques dans la région d'Izarène (Nord du Maroc). J Appl. Biosci 2015;86:7940-7956. 